LA-UR-02-3291

\title{
An Analytic Model of the Shear Modulus at All Temperatures and Densities
}

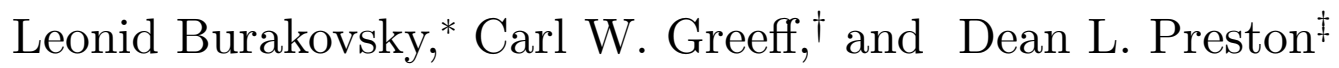 \\ Los Alamos National Laboratory \\ Los Alamos, NM 87545, USA
}

\begin{abstract}
An analytic model of the shear modulus applicable at temperatures up to melt and at all densities is presented. It is based in part on a relation between the melting temperature and the shear modulus at melt. Experimental data on argon are shown to agree with this relation to within $1 \%$. The model of the shear modulus involves seven parameters, all of which can be determined from zero-pressure experimental data. We obtain the values of these parameters for 11 elemental solids. Both the experimental data on the room-temperature shear modulus of argon to compressions of $\sim 2.5$, and theoretical calculations of the zero-temperature shear modulus of aluminum to compressions of $\sim 3.5$ are in good agreement with the model. Electronic structure calculations of the shear moduli of copper and gold to compressions of 2 , performed by us, agree with the model to within uncertainties.
\end{abstract}

Key words: dislocation, density, electronic structure, melting, pressure, shear modulus PACS: 61.72.Lk, 62.20.Dc, 62.50.+p, 64.10.+h, 64.70.Dv, 71.20.Be

\section{Introduction}

A reliable model of the adiabatic (isentropic) shear modulus, $G$, of a polycrystalline solid at temperatures to $T_{m}$, the melting temperature, and up to megabar pressures is needed for

${ }^{*}$ E-mail: BURAKOV@LANL.GOV

${ }^{\dagger}$ E-mail: GREEFF@LANL.GOV

${ }^{\ddagger}$ E-mail: DEAN@LANL.GOV 
many applications, including the modeling of plastic deformation at extremes of pressure and temperature, numerical calculations of elastic and shock wave propagation, and even calculations of the oscillations of low-mass astrophysical objects.

Adiabatic elastic properties are generally determined by ultrasonic wave-speed measurements, which are usually made in the low-pressure regime. Zero-pressure experimental data have been accumulated on single-crystal elastic constants, together with polycrystalline averages, at temperatures from $T=0$ to nearly $T_{m}$ for $\mathrm{Ag}$ (to within $60^{\circ} \mathrm{K}$ of $T_{m}$ ) [1], $\mathrm{Au}$ (to within $60^{\circ} \mathrm{K}$ of $T_{m}$ ) [2], Ge (to within $90^{\circ} \mathrm{K}$ of $T_{m}$ ) [3], and $\mathrm{V}$ (to within $80^{\circ} \mathrm{K}$ of $T_{m}$ ) [⿶]. The data run from $T=0$ to $T_{m}$ for $\mathrm{Al}$ [5], $\mathrm{Ar}$ [6], $\mathrm{Bi}$ [7], $\mathrm{Cd}$ [8], $\mathrm{Cs}$ [9], $\mathrm{Cu}$ [10], In [11], $\mathrm{K}$ |12], $\mathrm{Na}$ [13], $\mathrm{Nb}$ [14], $\mathrm{Ne}$ [15], $\mathrm{Pb}$ [11], Sn [7], Ta [16], Te [17], Xe [18], and $\mathrm{Zn} 19$.

On the theoretical side, it is possible to calculate singe-crystal elastic constants as a function of compression at zero temperature from electronic-structure theory. Such calculations were done by Straub et al. [20] for $\mathrm{Cu}$, Christensen et al. [21] for Mo and W, Söderlind et al. [22] for Fe, and Söderlind and Moriarty [23 for Ta. With known interatomic potentials it is possible to calculate the temperature dependence of the elastic constants by computer simulation techniques, as demonstrated by the calculations for $\mathrm{Na}$ [24], $\mathrm{Mg}$ [25], and $\mathrm{Cu}$ [26]. Bounds on the shear modulus, $G$, can be calculated from the single-crystal elastic constants for any crystal class [27], and for a cubic crystal the polycrystalline shear modulus can be calculated exactly using the Kröner cubic equation 28.

Guinan and Steinberg 29] modeled the zero-temperature shear modulus as $G=G_{0}+$ $G_{0}^{\prime} P\left(\rho_{0} / \rho\right)^{1 / 3}$, where $G_{0}^{\prime}$ is the pressure derivative of $G$ at zero pressure and $\rho$ is density. This functional form was chosen so that $G \sim \rho^{4 / 3}$ as $\rho \rightarrow \infty$, the correct asymptotic behavior albeit with a prefactor which does not generally coincide with that given by the one-component plasma model for $G$. Preston and Wallace [30] proposed a model for the temperature dependence of the shear modulus at any density, but left the density dependence itself arbitrary. The dependence of the shear modulus on both density and temperature has also been discussed by Anderson [31].

In this paper we develop an analytic model for the density and temperature dependence of the shear modulus by combining four key elements. First is a simple but accurate relation between the density, the melting temperature as a function of density, $T_{m}(\rho)$, and the shear modulus along the solidus 32, 33. Second is the Preston-Wallace model for the shear modulus. Third is an analytic model for the Gruneisen parameter [34 that is used in conjunction with the fourth ingredient, the Lindemann criterion [35], to generate an analytic expression for $T_{m}(\rho)$.

\section{A relation between shear modulus and melting temperature}

The melting temperature and shear modulus along the solidus approximately satisfy the relation

$$
\frac{G\left(\rho, T_{m}(\rho)\right)}{\rho T_{m}(\rho)}=\frac{G\left(\rho_{\mathrm{ref}}, T_{m}\left(\rho_{\mathrm{ref}}\right)\right)}{\rho_{\mathrm{ref}} T_{m}\left(\rho_{\mathrm{ref}}\right)}
$$


where $\rho_{\text {ref }}$ is a reference density. This relation is the foundation of our model for the shear modulus, so we provide theoretical justification for it following two approaches: the theory of dislocation-mediated melting [32, 33], and the theory of a Debye solid (in which it derives as a consequence of the proportionality of $G$ to the square of the Debye temperature). The relation is shown to agree very well with shear modulus data on argon, the only data available for such a comparison.

\subsection{Two derivations of relation (1)}

It follows from our model of melting as a dislocation-mediated phase transition that the relation

$$
k_{B} T_{m}=\frac{1-\nu\left(T_{m}\right) / 2}{1-\nu\left(T_{m}\right)} \frac{G\left(T_{m}\right) v\left(T_{m}\right)}{\ln (z-1)} \frac{\lambda}{8 \pi} \ln \left(\frac{\alpha^{2}}{4 b^{2} d\left(T_{m}\right)}\right) .
$$

holds at any pressure. Here $b$ is the magnitude of the Burgers vector, $\nu$ is the Poisson ratio, $v$ is the Wigner-Seitz volume, $\lambda \equiv b^{3} / v$ is a geometric factor characterizing the lattice, $z$ is the coordination number, and $d\left(T_{m}\right)$ is the dislocation density at melt. Note that the factors $\lambda$ and $\ln (z-1)$ explicitly account for the influence of crystal structure on melting. The value of $\lambda$ is $3 \sqrt{3} / 4 \approx 1.30$ for body-centered cubic (bcc), and $\sqrt{2} \approx 1.41$ for face-centered cubic (fcc) and ideal $(c / a=\sqrt{8 / 3})$ hexagonal close-packed (hcp) lattices [33. The parameter $\alpha$ is the ratio of $b$ to the dislocation core radius, $r_{0} ; \alpha \approx 2.9$ for both bcc and fcc crystals [33]. This melting relation plus experimental data on over half the elements in the periodic table give $b^{2} d\left(T_{m}\right)=0.61 \pm 0.20$ (throughout this paper the error in such expressions is the corresponding standard deviation) with $G\left(300^{\circ} \mathrm{K}\right), v_{W S}\left(300^{\circ} \mathrm{K}\right)$ used instead of $G\left(T_{m}\right), v_{W S}\left(T_{m}\right)$, respectively [32].

\begin{tabular}{|l|l|l|l|l|l|l|l|l|l|l|l|}
\hline element & $\mathrm{Ba}$ & $\mathrm{Cs}$ & $\mathrm{Cr}$ & $\delta$-Fe & $\mathrm{K}$ & $\mathrm{Li}$ & $\mathrm{Na}$ & $\mathrm{Nb}$ & $\mathrm{Rb}$ & $\beta$-Ti & $\mathrm{V}$ \\
\hline$T_{m},{ }^{\circ} \mathrm{K}$ & 1000 & 301.6 & 2130 & 1811 & 336.5 & 453.7 & 370.9 & 2750 & 312.5 & 1941 & 2183 \\
\hline$v\left(T_{m}\right), \mathrm{A}^{3}$ & 66.68 & 116.8 & 13.10 & 12.76 & 76.38 & 22.14 & 40.17 & 19.33 & 93.37 & 18.61 & 14.89 \\
\hline$G\left(T_{m}\right), \mathrm{GPa}$ & 2.96 & 0.39 & 35.7 & 30.8 & 0.80 & 3.60 & 1.93 & 32.6 & 0.60 & 21.9 & 32.3 \\
\hline$G v /\left(k_{B} T_{m}\right)$ & 14.3 & 10.9 & 15.9 & 15.7 & 13.2 & 12.7 & 15.1 & 16.6 & 13.0 & 15.2 & 15.9 \\
\hline
\end{tabular}

Table 1. Numerical values of the ratio $G\left(T_{m}\right) v\left(T_{m}\right) /\left(k_{B} T_{m}\right)$ for 11 elemental solids that melt from bcc crystalline structure at normal pressure.

\begin{tabular}{|l|l|l|l|l|l|l|l|l|l|l|l|}
\hline element & $\mathrm{Ag}$ & $\mathrm{Al}$ & $\mathrm{Ar}$ & $\mathrm{Au}$ & $\beta$-Co & $\mathrm{Cu}$ & $\mathrm{Ni}$ & $\mathrm{Pb}$ & $\mathrm{Pd}$ & $\mathrm{Pt}$ & $\mathrm{Rh}$ \\
\hline$T_{m},{ }^{\circ} \mathrm{K}$ & 1235 & 933.5 & 83.8 & 1338 & 1768 & 1358 & 1728 & 600.6 & 1828 & 2041 & 2237 \\
\hline$v\left(T_{m}\right), \mathrm{A}^{3}$ & 18.19 & 17.55 & 40.90 & 17.88 & 11.96 & 12.61 & 11.85 & 31.14 & 15.65 & 16.04 & 14.87 \\
\hline$G\left(T_{m}\right), \mathrm{GPa}$ & 17.2 & 15.6 & 0.60 & 15.2 & 34.7 & 27.1 & 38.6 & 5.60 & 35.0 & 32.0 & 55.0 \\
\hline$G v /\left(k_{B} T_{m}\right)$ & 18.4 & 21.2 & 21.2 & 14.7 & 17.0 & 18.2 & 19.2 & 21.0 & 21.7 & 18.2 & 26.5 \\
\hline
\end{tabular}

Table 2. Numerical values of the ratio $G\left(T_{m}\right) v\left(T_{m}\right) /\left(k_{B} T_{m}\right)$ for 11 elemental solids that melt from fcc crystalline structure at normal pressure. 
From the compilation of data in Tables 1 and 2 we find that the product of $\lambda$ and the logarithm in Eq. (2) (with $\nu\left(T_{m}\right)=0.42 \pm 0.02$ [36]) is a constant to $15 \%$ at zero pressure:

$$
\frac{\lambda}{8 \pi} \ln \left(\frac{\alpha^{2}}{4 b^{2} d\left(T_{m}\right)}\right)=\left[\begin{array}{ll}
0.100 \pm 0.015, & \text { bcc, } \\
0.091 \pm 0.014, & \text { fcc. }
\end{array}\right.
$$

We make the reasonable assumption that the mean interdislocation distance at the melting point, $2 R \approx 1 / \sqrt{d\left(T_{m}\right)}$, scales with $b$, which implies that $b^{2} d\left(T_{m}\right)$ is a compressionindependent constant. It is also assumed that $\alpha^{-1}=r_{0} / b$ is unchanged under compression. Hence $\lambda \ln \left(\alpha^{2} / 4 b^{2} d\right)$ is expected to be pressure-independent with approximately the same value for both bcc and fcc elements. It then follows from (2) that for a given element

$$
\xi(P) \equiv \frac{1-\nu\left(P, T_{m}(P)\right) / 2}{1-\nu\left(P, T_{m}(P)\right)} \frac{G\left(P, T_{m}(P)\right) v\left(P, T_{m}(P)\right)}{k_{B} T_{m}(P) \ln (z-1)}=c,
$$

where the constant $c$ has nearly the same value for both bcc and fcc elements. Experimental validation of this relation is not posible because of a lack of data from moderate to high compressions. However, the $P \rightarrow 0$ and $P \rightarrow \infty$ limits are consistent with Eq. (4), which we now demonstrate.

At very high compressions a solid becomes a crystallized one-component plasma (OCP), i.e., a lattice of ions in a uniform neutralizing background of electrons [35]. The melting curve of a solid at ultrahigh pressures is described by the equation

$$
\frac{Z^{2} e^{2}}{a\left(T_{m}\right) k_{B} T_{m}}=\Gamma_{m}
$$

where $Z$ is the atomic number, $a=(3 v / 4 \pi)^{1 / 3}$ is the Wigner-Seitz radius, and $\Gamma_{m}$, a dimensionless constant, is the OCP coupling parameter at melt [35]. Numerous calculations of $\Gamma_{m}$ for a bcc OCP crystal (see ref. [37] for a review) converge on the value 175 [38, 39]. The value of $\Gamma_{m}$ for a fcc OCP crystal has been calculated to be $196 \pm 1$ [40] and 208.3 [11; hence we take $\Gamma_{m}=200$ for a fcc OCP crystal in the following analysis. The bcc OCP single-crystal elastic constants $\left(c_{11}-c_{12}\right) / 2$ and $c_{44}$ have been calculated by means of Monte-Carlo simulations [42]. A linear fit to the values of $G$ given by the formula of Sisodia et al. 433 (when $c_{11}$ and $c_{12}$ are not known separately, the value of $G$ given by this formula approximates Kröner's shear modulus with high accuracy and, in fact, tends to the precise Kröner value in the limit $P \rightarrow \infty$ ) results in [37]

$$
G_{\mathrm{bcc}}^{\mathrm{OCP}}(T)=g_{\mathrm{bcc}}\left(\frac{4 \pi}{3}\right)^{1 / 3} \frac{Z^{2} e^{2}}{v^{4 / 3}}\left(1-\beta_{\mathrm{bcc}}^{\mathrm{OCP}} \frac{T}{T_{m}}\right),
$$

where $g_{\mathrm{bcc}}=0.09301$ and $\beta_{\mathrm{bcc}}^{\mathrm{OCP}}=0.21 \pm 0.18$. We have calculated (unpublished) the coefficient $g_{\mathrm{fcc}}$ to be 0.09011 . The coefficient $\beta_{\mathrm{fcc}}^{\mathrm{OCP}}$ has not been calculated, so we assume $\beta_{\mathrm{fcc}}^{\mathrm{OCP}}=\beta_{\mathrm{bcc}}^{\mathrm{OCP}}$. We have also calculated the Voigt $(\mathrm{V})$ and Reuss $(\mathrm{R})$ bounds on the shear modulus of an ideal hcp OCP crystal: $g_{\mathrm{hcp}}^{\mathrm{V}}=0.1194, g_{\mathrm{hcp}}^{\mathrm{R}}=0.1045$, hence $g_{\mathrm{hcp}}=0.1120$ for the Voigt-Reuss-Hill average.

From Eqs. (4) and (6), and the ultrahigh pressure limit $\nu(T)=1 / 2$ [44, 45], we obtain $\xi_{\mathrm{bcc}}^{\mathrm{OCP}}=9.9 \pm 2.3$ and $\xi_{\mathrm{fcc}}^{\mathrm{OCP}}=8.9 \pm 2.0$. Comparison of the $\mathrm{OCP}$ values of $\xi$ to their 
zero-pressure counterparts (which follow from Eqs. (2) and (3)), $\xi_{\text {bcc }}(0)=10.0 \pm 1.5$ and $\xi_{\text {fcc }}(0)=11.0 \pm 1.7$, shows that the $P=0$ and $\mathrm{OCP}$ values agree to within uncertainties, compelling evidence, though not a proof, that Eq. (4) is in fact valid, at least for bcc and fcc lattices. The uncertainty-weighted average of the four values is $10.0 \pm 1.8$.

Formula (1) now follows from Eq. (4) provided that the ratio $\left(1-\nu\left(T_{m}\right) / 2\right) /\left(1-\nu\left(T_{m}\right)\right)$ is (approximately) a constant; in fact this ratio varies between $\approx 4 / 3$ at $P=0$ and $3 / 2$ as $P \rightarrow \infty$, i.e., it is $(17 \pm 1) / 12 \approx 17 / 12$ to $94 \%$ accuracy.

Formula (1) can also be derived from the theory of a Debye solid. Ledbetter 46. derived the Debye-solid relation

$$
\Theta_{D}=\frac{\Lambda}{v^{1 / 3}} \sqrt{\frac{G}{\rho}},
$$

where $\Theta_{D}$ is the Debye temperature and $\Lambda$ is a constant. (Since $G \sim \rho^{4 / 3}$ as $\rho \rightarrow \infty$, $\Theta_{D} \sim \rho^{1 / 2}$, which is consistent with $\gamma$ (Grüneisen) $\rightarrow 1 / 2$ [34, 44]. Its widely used counterpart 46], $\Theta_{D}=\tilde{\Lambda} v^{-1 / 3} \sqrt{B / \rho}$, where $B$ is the bulk modulus, has the wrong asymptotic behavior, $\Theta_{D} \sim \rho^{2 / 3}$.) Siethoff and Ahlborn [47] demonstrated the validity of the Ledbetter formula at $P=0$ for Debye-like cubic solids [47, 48, 49], non-Debye hexagonal and tetragonal solids [50], and intermetallic compounds [51]. Eq. (7), $v \sim 1 / \rho$, and the Lindemann melting criterion [35]

$$
\frac{T_{m}(\rho) \rho^{2 / 3}}{\Theta_{D}^{2}(\rho)}=\mathrm{constant}
$$

again yield relation (1).

\begin{tabular}{|r|c|c|c|c|}
\hline$T_{m}(P),{ }^{\circ} \mathrm{K}$ & $v\left(P, T_{m}(P)\right), \mathrm{\AA}^{3}$ & $u_{t}, \mathrm{~m} / \mathrm{s}$ & $G\left(P, T_{m}(P)\right), \mathrm{GPa}$ & $G v /\left(k_{B} T_{m}\right)$ \\
\hline 205.59 & 35.698 & 952.6 & 1.686 & 21.21 \\
190.90 & 36.216 & 909.7 & 1.516 & 20.84 \\
175.91 & 36.785 & 879.5 & 1.395 & 21.14 \\
162.80 & 37.319 & 843.0 & 1.263 & 20.98 \\
162.07 & 37.350 & 847.0 & 1.274 & 21.28 \\
148.19 & 37.959 & 800.0 & 1.118 & 20.75 \\
134.47 & 38.601 & 768.6 & 1.015 & 21.11 \\
123.16 & 39.155 & 736.0 & 0.918 & 21.15 \\
\hline 83.80 & 40.900 & & 0.600 & 21.22 \\
\hline
\end{tabular}

Table 3. Numerical values of the ratio $G\left(P, T_{m}(P)\right) v\left(P, T_{m}(P)\right) /\left(k_{B} T_{m}(P)\right)$ for Ar along its solidus. The last row of the table contains $P=0$ values.

\subsection{Experimental verification}

Direct experimental validation of relation (1) over a restricted range of densities is possible for a single element, viz. argon. Ishizaki et al. [52 measured the transverse ultrasonic wave velocity, $u_{t}$, in compressed argon along its solidus as a function of temperature. 
We calculate the shear modulus from the formula $u_{t}=\sqrt{G / \rho}$, and $v=V / N_{A}$ from the measured argon melting curve [53], $V=V\left(T_{m}\right), V$ being the molar volume. Our results for the values of $G v /\left(k_{B} T_{m}\right)$ are shown in Table 3.

For the $P>0$ data we find $G v /\left(k_{B} T_{m}\right)=21.06 \pm 0.17$, in agreement with its $P=0$ value (we get $G v /\left(k_{B} T_{m}\right)=21.08 \pm 0.17$ for all of the data). Thus, $G v /\left(k_{B} T_{m}\right)$ for $\operatorname{Ar}$ deviates from a constant by less than $1 \%$.

\section{Model of the shear modulus at all temperatures and densities}

Preston and Wallace [30] constructed a model of the temperature dependence of the shear modulus $\left(0 \leq T \leq T_{m}\right)$ for arbitrary pressures. The $T$-dependence of $G$ involves two characteristic temperatures, namely the Debye temperature and the melting temperature. The shear modulus is always monotonically decreasing with $T$, and is nonlinear for $T \lesssim \Theta_{D}$ and linear from $\Theta_{D}$ to $T_{m}$ for most elements. An accurate representation of $G(T)$ at fixed density is achieved by ignoring the low-temperature non-linearity and approximating $G(T)$ as a linear function of the reduced temperature $T / T_{m}$ with the correct value $G(\rho, 0)$ at $T=0$ 30:

$$
G(\rho, T)=G(\rho, 0)\left(1-\beta \frac{T}{T_{m}(\rho)}\right),
$$

In general, the parameter $\beta$ may be density dependent. A fit to shear-modulus data spanning temperatures from $T=0$ to $T / T_{m} \gtrsim 0.4$ at zero pressure gave $\beta_{0}=0.23 \pm 0.08$ [30]. (For the $11 \mathrm{fcc}$ elements in Table 4 below $\beta_{0}=0.27 \pm 0.10$.) On the other hand, $\beta^{\mathrm{OCP}}=0.21 \pm 0.18$ (as discussed above), which equals $\beta_{0}$ to within uncertainties, so we assume that $\beta$ is independent of density. At $\rho=\rho_{\text {ref }}$ and $T=T_{m}\left(\rho_{\text {ref }}\right)$, Eq. (9) reduces to

$$
\beta=1-\frac{G\left(\rho_{\mathrm{ref}}, T_{m}\left(\rho_{\mathrm{ref}}\right)\right)}{G\left(\rho_{\mathrm{ref}}, 0\right)} .
$$

The linear temperature dependence is suggested by available $P=0$ experimental data on $G$ over the temperature range $0 \leq T \leq T_{m}$ [1-19]. This straight-line representation turns out to be quite accurate: the maximum deviation of the data from the corresponding fitted lines is $\sim 5 \%$ for 21 of the 22 metals analyzed in 30. The exception is uranium, for which $G(T)$ is nonlinear throughout the $\alpha$ phase at $P=0$. As mentioned above, $G(T)$ is is nonlinear below $\Theta_{D}$, thus $G(T)$ is nonlinear for low-melting-point solids from $T=0$ to $T_{m}$. Despite the nonlinearity of $G(T)$ in these cases, the model uncertainty is only of order $10 \%$.

At any given pressure, the introduction of the temperature dependence of the density, $\rho=\rho(T)$, into Eq. (9) gives the temperature dependence of $G$ at that pressure. In Fig. 1 we compare $G(\rho(T), T)$ for $0 \leq T \leq T_{m}$ at $P=0$ for $\mathrm{Au}$ and $\mathrm{Cu}$ to experimental data [2, 10]. The temperature dependence of the density was taken from ref. [54, and $G(\rho, 0)$ and $T_{m}(\rho)$ are described by Eqs. (13) and (14) below with parameter values from Tables 2 and 4. 


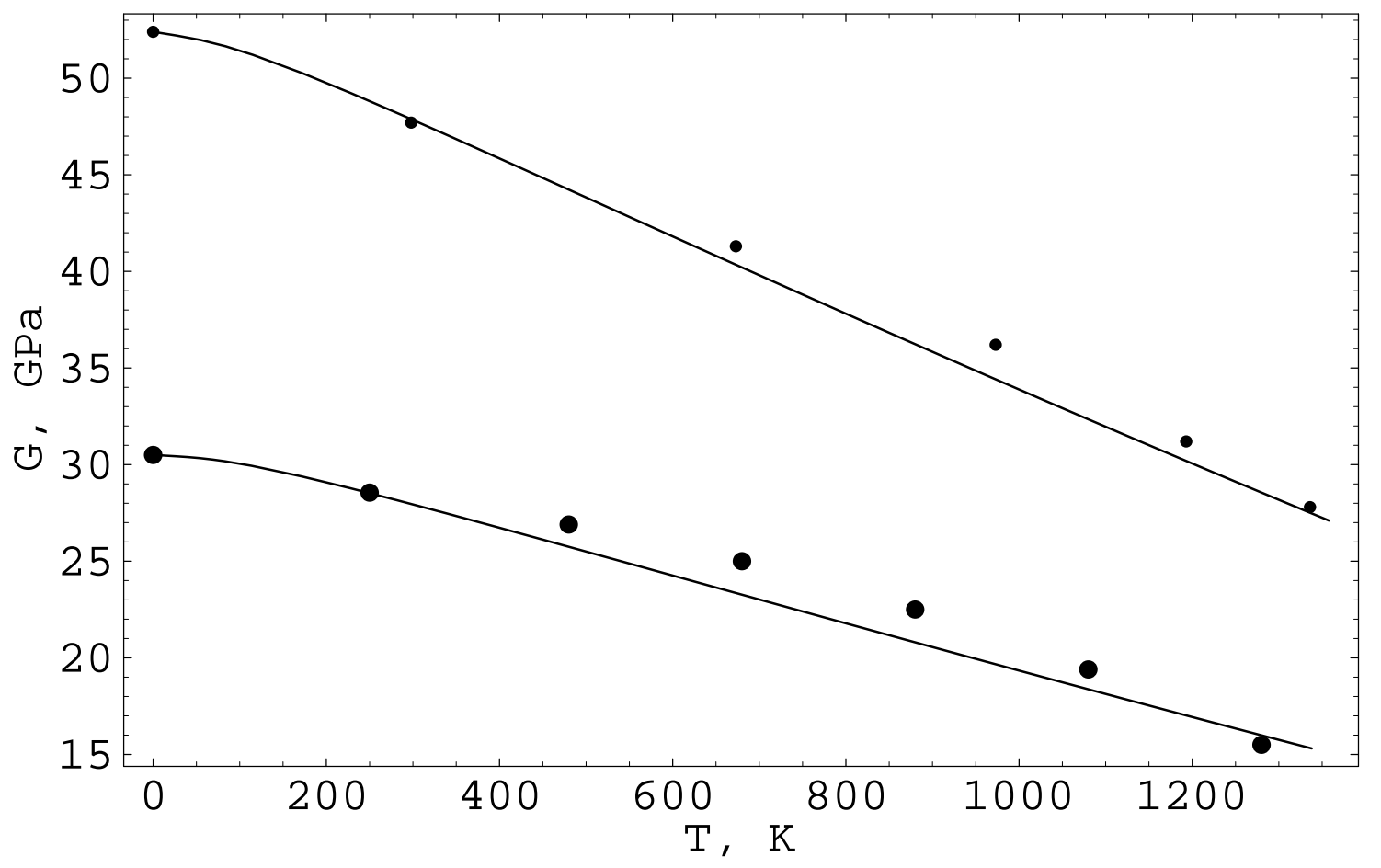

Fig. 1. The $P=0$ shear moduli of $\mathrm{Cu}$ and $\mathrm{Au}$ : Eq. (9) with $\rho=\rho(T)$ from ref. 554 and $G(\rho(T), 0)$ and $T_{m}(\rho(T))$ described by Eqs. (13) and (14) with the parameters from Tables 2 and 4 vs. the experimental data on $\mathrm{Cu}$ [10] (smaller points) and $\mathrm{Au}$ [2] (larger points).

The Grüneisen parameter was recently modeled as 34

$$
\gamma(\rho)=\frac{1}{2}+\frac{\gamma_{1}}{\rho^{1 / 3}}+\frac{\gamma_{2}}{\rho^{q}}, \quad \gamma_{1}, \gamma_{2}, q=\text { const, } q>1
$$

through consideration of its low- and ultrahigh-pressure limits. This analytic form for $\gamma$ was obtained under the assumptions that (i) $\gamma \rightarrow 1 / 2$ as $\rho \rightarrow \infty$, (ii) $\gamma$ is an analytic function of $x \equiv 1 / \rho^{1 / 3}$, essentially the interatomic distance, and (iii) the coefficient of $x$ in the Taylor-Maclaurin series expansion for $\gamma$ is non-zero. The third term on the righthand- side of Eq. (11) represents the contribution of the quadratic and higher-order terms in the power series. The procedure for calculating the values of $\gamma_{1}, \gamma_{2}$, and $q$ is discussed below.

Eq. (11) and the Lindemann criterion [35]

$$
\frac{d \ln T_{m}(\rho)}{d \ln \rho}=2\left(\gamma(\rho)-\frac{1}{3}\right)
$$

provide a model for the density dependence of the melting temperature,

$$
T_{m}(\rho)=T_{m}\left(\rho_{\mathrm{ref}}\right)\left(\frac{\rho}{\rho_{\mathrm{ref}}}\right)^{1 / 3} \exp \left\{6 \gamma_{1}\left(\frac{1}{\left(\rho_{\mathrm{ref}}\right)^{1 / 3}}-\frac{1}{\rho^{1 / 3}}\right)+\frac{2 \gamma_{2}}{q}\left(\frac{1}{\left(\rho_{\mathrm{ref}}\right)^{q}}-\frac{1}{\rho^{q}}\right)\right\} .
$$


The natural choice for the reference density is $\rho_{m}$, the zero-pressure density at melt, which is known experimentally in most cases (see, e.g., [54]).

Finally, Eqs. (1),(9),(10), and (13) result in

$$
G(\rho, 0)=G\left(\rho_{\mathrm{ref}}, 0\right)\left(\frac{\rho}{\rho_{\mathrm{ref}}}\right)^{4 / 3} \exp \left\{6 \gamma_{1}\left(\frac{1}{\left(\rho_{\mathrm{ref}}\right)^{1 / 3}}-\frac{1}{\rho^{1 / 3}}\right)+\frac{2 \gamma_{2}}{q}\left(\frac{1}{\left(\rho_{\mathrm{ref}}\right)^{q}}-\frac{1}{\rho^{q}}\right)\right\}
$$

where $\rho_{\text {ref }}$ is most conveniently chosen to be either $\rho_{m}$ or $\rho_{0}$, the density at zero pressure and temperature.

Eqs. (9),(13), and (14) constitute our analytic model for the shear modulus. It requires the determination of 7 parameters, namely $\rho_{\text {ref }}, G\left(\rho_{\text {ref }}, 0\right), T_{m}\left(\rho_{\text {ref }}\right), \gamma_{1}, \gamma_{2}, q$, and $\beta$. The values of $\gamma_{1}, \gamma_{2}$ and $q$ are obtained by simultaneous solution of Eq. (11) with $\rho=$ $\rho\left(T=300^{\circ} \mathrm{K}\right)$ and $\rho=\rho_{m}$, and Eq. (5) with $\Gamma_{m}=180$ 34 and $T_{m}(\rho)$ given by the high-density limit of Eq. (13). The value of $\gamma\left(\rho_{m}\right)$ is obtained from the Kraut-Kennedy relation [55] and low-pressure melting data. The remaining parameters are either zeropressure experimental data themselves or can be determined from such data (for example, $\beta)$. In Table 4 we present the values of $\rho_{\text {ref }}$ (both $\rho_{0}$ and $\left.\rho_{m}\right), G\left(\rho_{0}, 0\right), \gamma_{1}, \gamma_{2}, q$, and $\beta$ for all of the fcc elements of Table 2. The values of $G\left(\rho_{m}, 0\right)$ can be calculated from the relation $G\left(\rho_{m}, 0\right)=G\left(\rho_{m}, T_{m}\right) /(1-\beta)$ with $G\left(\rho_{m}, T_{m}\right)$ from Table 2, which also contains the values of $T_{m}\left(\rho_{m}\right)$. Since $\beta$-Co exists only above $T \approx 700^{\circ} \mathrm{K}$ at $P=0$, its values of $G\left(\rho_{0}, 0\right)$ and $\beta$ were obtained from the conditions $G\left(\rho_{m}, T_{m}\right)=34.7$ and $G\left(\rho\left(T=710^{\circ} \mathrm{K}\right)=8.62, T=710^{\circ} \mathrm{K}\right)=57.1$ [56].

\begin{tabular}{|r|c|c|c|c|l|c|c|}
\hline element & $\rho_{0}, \mathrm{~g} / \mathrm{cc}$ & $\rho_{m}, \mathrm{~g} / \mathrm{cc}$ & $G\left(\rho_{0}, 0\right), \mathrm{GPa}$ & $\gamma_{1},(\mathrm{~g} / \mathrm{cc})^{1 / 3}$ & $\gamma_{2},(\mathrm{~g} / \mathrm{cc})^{q}$ & $q$ & $\beta$ \\
\hline $\mathrm{Ag}$ & 10.63 & 9.850 & 33.5 & 2.23 & $9.63 \cdot 10^{4}$ & 4.8 & 0.18 \\
$\mathrm{Al}$ & 2.730 & 2.550 & 29.3 & 0.84 & 45.4 & 3.5 & 0.22 \\
$\mathrm{Ar}$ & 1.771 & 1.622 & 1.46 & 1.06 & 6.42 & 2.2 & 0.23 \\
$\mathrm{Au}$ & 19.49 & 18.29 & 30.5 & 3.21 & $1.97 \cdot 10^{12}$ & 9.4 & 0.18 \\
$\beta \mathrm{Co}$ & 8.910 & 8.180 & 73.2 & 1.81 & $6.28 \cdot 10^{4}$ & 5.5 & 0.33 \\
$\mathrm{Cu}$ & 9.020 & 8.370 & 52.4 & 1.87 & $2.31 \cdot 10^{4}$ & 4.7 & 0.25 \\
$\mathrm{Ni}$ & 8.970 & 8.220 & 93.6 & 1.85 & $5.60 \cdot 10^{5}$ & 6.5 & 0.41 \\
$\mathrm{~Pb}$ & 11.60 & 11.05 & 11.7 & 3.09 & $8.21 \cdot 10^{8}$ & 8.5 & 0.36 \\
$\mathrm{Pd}$ & 12.13 & 11.29 & 50.3 & 2.40 & $3.34 \cdot 10^{6}$ & 6.6 & 0.07 \\
$\mathrm{Pt}$ & 21.58 & 20.19 & 66.3 & 3.21 & $1.13 \cdot 10^{11}$ & 8.3 & 0.27 \\
$\mathrm{Rh}$ & 12.49 & 11.49 & 158. & 2.16 & $1.46 \cdot 10^{7}$ & 6.5 & 0.42 \\
\hline
\end{tabular}

Table 4. Numerical values of the model parameters for 11 fcc elements. The corresponding values of $T_{m}\left(\rho_{m}\right)$ and $G\left(\rho_{m}, T_{m}\left(\rho_{m}\right)\right)$ are provided in Table 2.

In Figs. 2, 3, and 4 we compare the melting curves of $\mathrm{Ar}, \mathrm{Al}$ and $\mathrm{Cu}$ as given by Eq. (13) with the corresponding parameters from Table 4 to experimental data. 


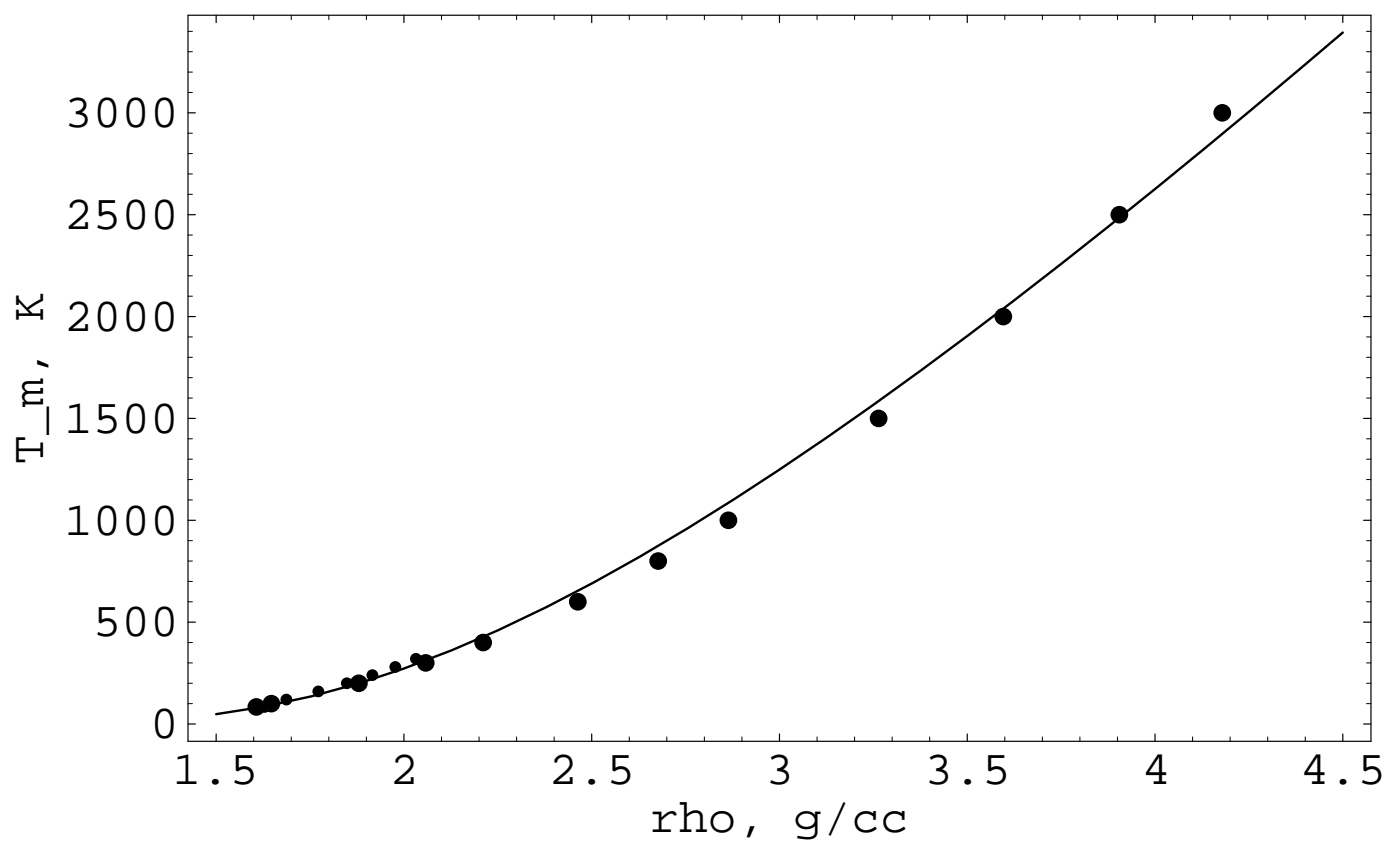

Fig. 2. Melting curve of Ar: Eq. (13) with the Ar parameters from Table 4 vs. data. The smaller points are the experimental data of ref. [53], and the larger points are the results of calculations [57].

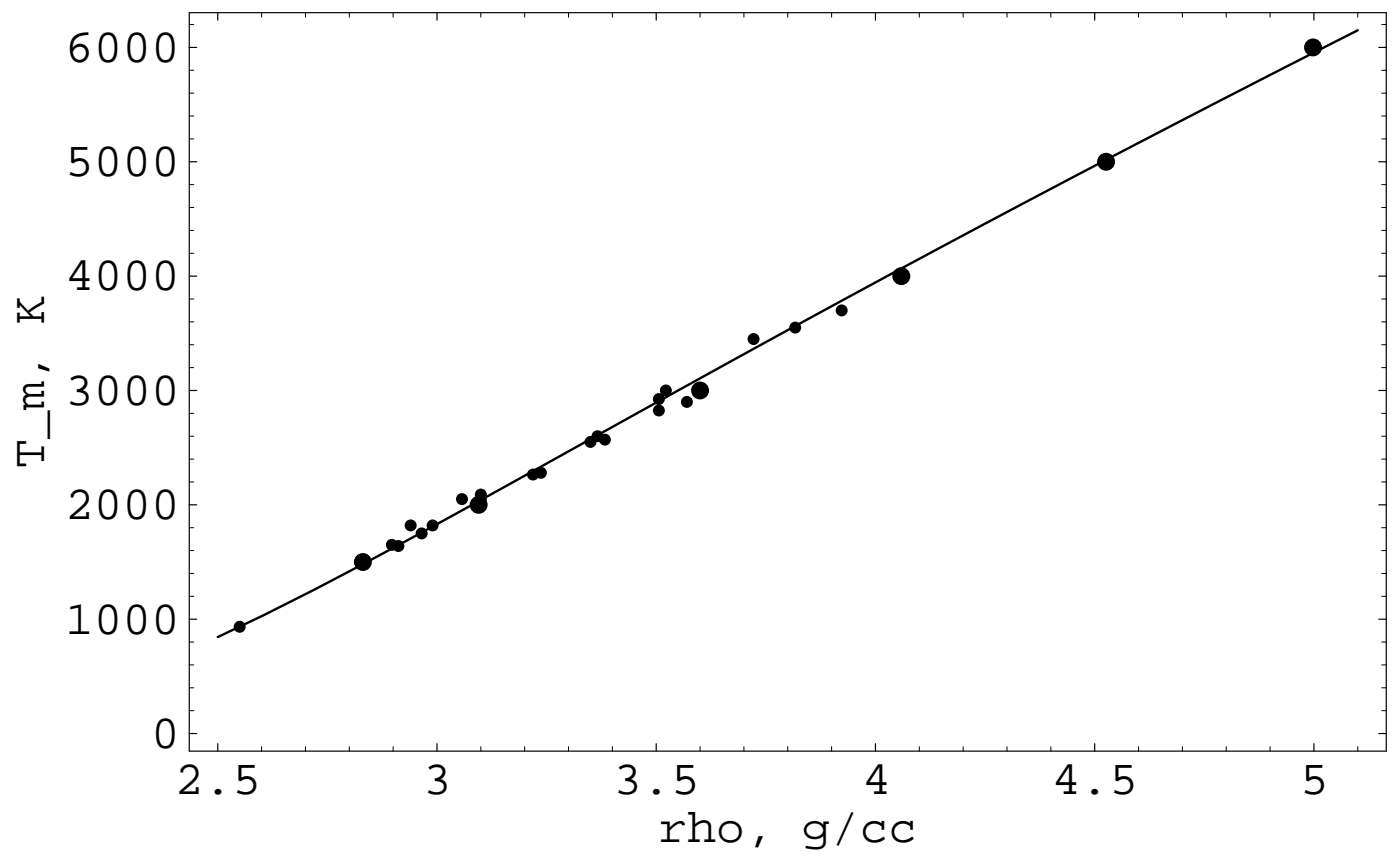

Fig. 3. Melting curve of Al: Eq. (13) with the Al parameters from Table 4 vs. data. The smaller points are the experimental data of ref. [58], and the larger points are the results of calculations [59]. 


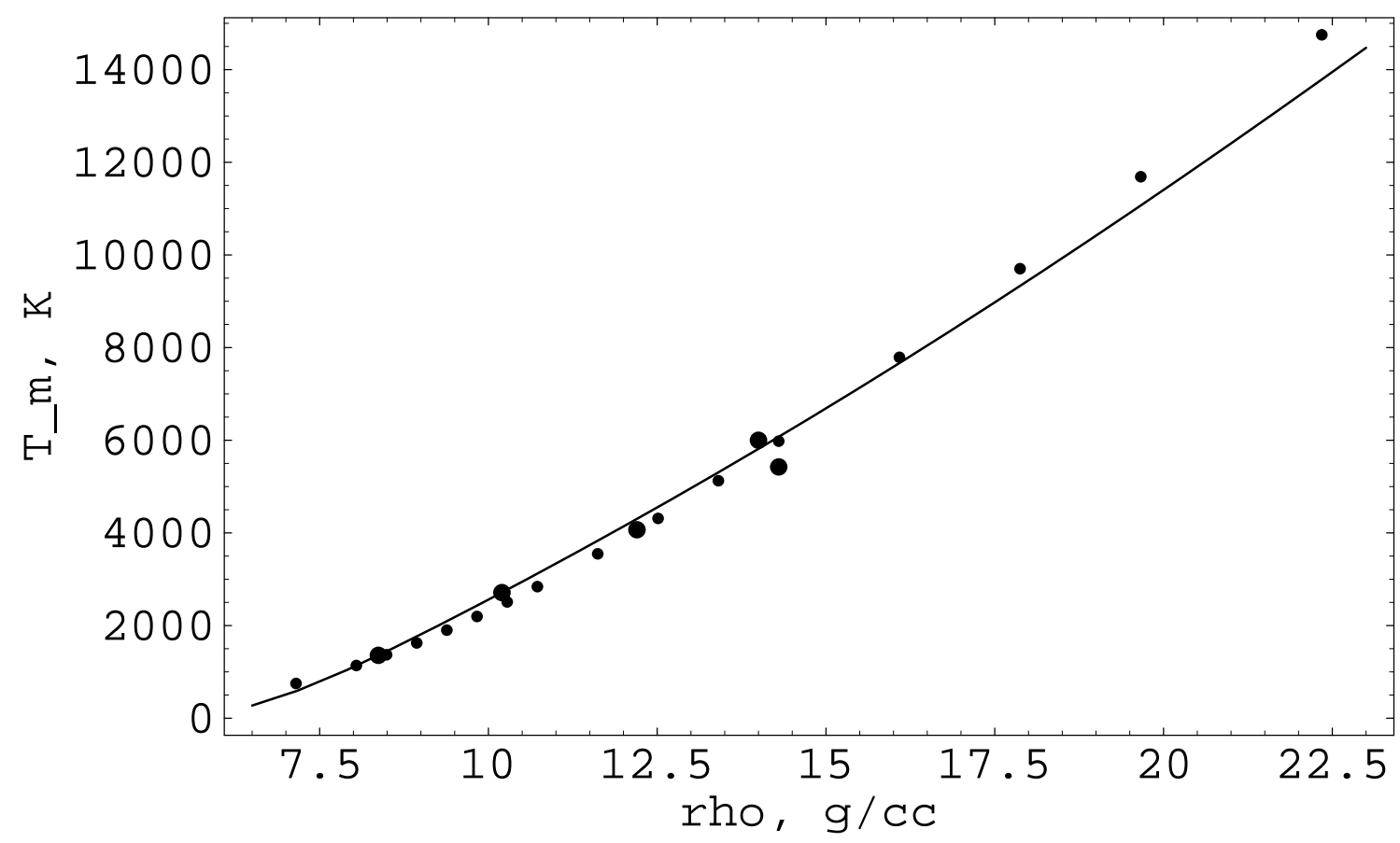

Fig. 4. Melting curve of Cu: Eq. (13) with the $\mathrm{Cu}$ parameters from Table 4 vs. data. The smaller points are from a new SESAME melting curve table for $\mathrm{Cu}$ [60]. The larger points are the $P=0$ reference point at (in $\mathrm{g} / \mathrm{cc}$ ) $\rho=8.4$, and the shock-melting points of ref. [61] at $\rho=10.2,12.2$ and 14.3, and of refs. [62, 63] at $\rho=14.0$.

Only five of the seven shear modulus parameters are independent because four appear in the model as two ratios, namely $\beta / T_{m}(\rho)$ in Eq. (9) and $G\left(\rho_{\text {ref }}, 0\right) /\left(\rho_{\text {ref }}\right)^{4 / 3}$ in Eq. (14); hence the shear modulus is of the form

$$
G(\rho, T)=a_{1} \rho^{4 / 3} \exp \left\{-\frac{a_{2}}{\rho^{q}}-\frac{a_{3}}{\rho^{1 / 3}}\right\}-a_{4} \rho T, \quad a_{1}, a_{2}, a_{3}, a_{4}, q=\text { const }>0 .
$$

As specific examples we provide the following formulas for the shear moduli of $\mathrm{Ar}, \mathrm{Al}$, $\mathrm{Cu}$, and $\mathrm{Au}$ :

$$
\begin{gathered}
G_{\mathrm{Ar}}(\rho, T)=687.4 \rho^{4 / 3} \exp \left\{-\frac{5.84}{\rho^{2.2}}-\frac{6.36}{\rho^{1 / 3}}\right\}-1.32 \cdot 10^{-3} \rho T \\
G_{\mathrm{Al}}(\rho, T)=611.8 \rho^{4 / 3} \exp \left\{-\frac{25.9}{\rho^{3.5}}-\frac{5.04}{\rho^{1 / 3}}\right\}-1.85 \cdot 10^{-3} \rho T \\
G_{\mathrm{Cu}}(\rho, T)=841.2 \rho^{4 / 3} \exp \left\{-\frac{9.83 \cdot 10^{3}}{\rho^{4.7}}-\frac{11.22}{\rho^{1 / 3}}\right\}-7.96 \cdot 10^{-4} \rho T \\
G_{\mathrm{Au}}(\rho, T)=1022.0 \rho^{4 / 3} \exp \left\{-\frac{4.19 \cdot 10^{11}}{\rho^{9.4}}-\frac{19.26}{\rho^{1 / 3}}\right\}-1.37 \cdot 10^{-4} \rho T .
\end{gathered}
$$




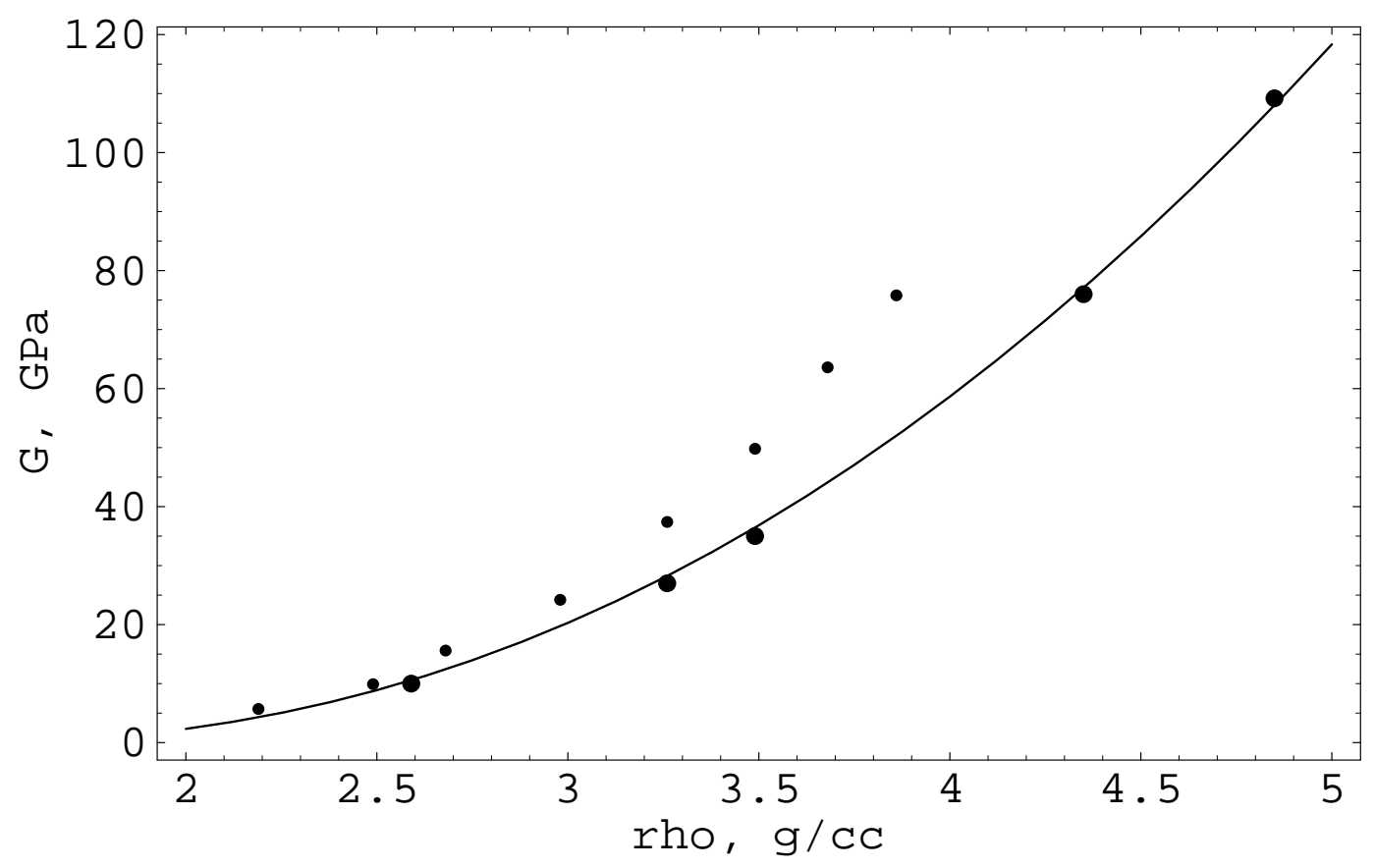

Fig. 5. The $T=300$ shear modulus of Ar: Eq. (16) vs. older 64 (smaller points) and more recent [65] (larger points) experimental data. The experimental technique used to obtain the older data has been criticized [65.

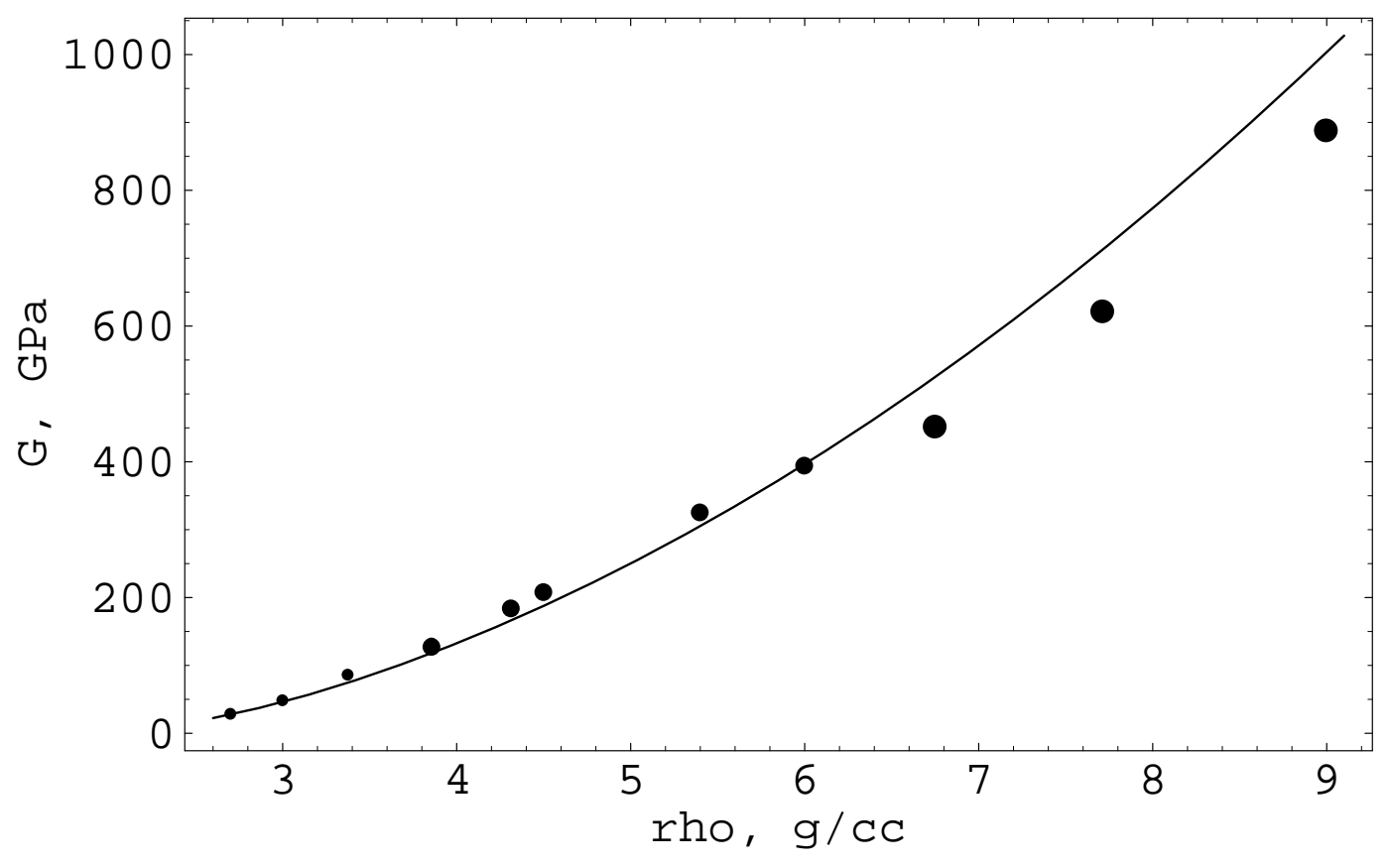

Fig. 6. The $T=0$ shear modulus of Al: Eq. (17) vs. the electronic-structure calculations of ref. [66]. The small, medium, and large points represent the values of $G$ in fcc, hcp, and bcc phases of $\mathrm{Al}$, respectively. 
In Fig. 5 we compare the $T=300^{\circ} \mathrm{K}$ shear modulus of $\mathrm{Ar}$ as given by Eq. (16) to experimental data. The $T=0$ shear modulus of $\mathrm{Al}$ from Eq. (17) is compared to the results of electronic structure calculations in Fig. 6 . The $T=0$ shear moduli of $\mathrm{Cu}$ and $\mathrm{Au}$ as given, respectively, by Eqs. (18) and (19) are compared to the results of the corresponding electronic-structure calculations in Figs. 7 and 8 in the next section.

\section{Comparison of model to electronic-structure re- sults for $\mathrm{Cu}$ and $\mathrm{Au}$}

With the exception of Ar, experimental data are not available to test the model to megabar pressures. We can, however, test the $T=0$ version of the model by comparing it to the results of $a b$ initio electronic-structure calculations of the shear modulus.

Electronic structure calculations based on approximate density functional theories have proven to give good predictions for a variety of material properties. A study of the elastic constants of several elements and compounds [67 covering a wide range of elastic properties, found errors with respect to experiment of generally less than $10 \%$ in the isotropic shear modulus. These results are obtained without empirical inputs. We expect such calculations to have similar accuracy under compression, thus providing a test of the new analytic model.

For this reason, we have carried out electronic structure calculations to obtain the single crystal elastic constants $C^{\prime}=\left(C_{11}-C_{12}\right) / 2, C_{44}$, and $B=\frac{1}{3}\left(C_{11}+2 C_{12}\right)$ for the fcc metals $\mathrm{Cu}$ and $\mathrm{Au}$ from normal to twice normal density. From these an average polycrystalline shear modulus is calculated and compared to the model.

The method for the calculations was described by Söderlind et al. 68. To evaluate $C^{\prime}$, the lattice is deformed by the (volume conserving) transformation

$$
\left(\begin{array}{ccc}
1+\delta & 0 & 0 \\
0 & 1+\delta & 0 \\
0 & 0 & 1 /(1+\delta)^{2}
\end{array}\right)
$$

The resulting energy change is

$$
\Delta E / V=6 C^{\prime} \delta^{2}+O\left(\delta^{3}\right)
$$

Similarly, $C_{44}$ is obtained by applying the (volume conserving) transformation

$$
\left(\begin{array}{ccc}
1 & \delta & 0 \\
\delta & 1 & 0 \\
0 & 0 & 1 /\left(1-\delta^{2}\right)
\end{array}\right),
$$

resulting in an energy change

$$
\Delta E / V=2 C_{44} \delta^{2}+O\left(\delta^{4}\right)
$$

In our calculations we have evaluated the energy as a function of $\delta$ at intervals of 0.01 , up to $\delta=0.04$. For the $C^{\prime}$ case the energy is not an even function of $\delta$, and so negative 
values of delta were used. The resulting $E(\delta)$ were fit to $4^{\text {th }}$ degree polynomials and the quadratic coefficent was used to evaluate the elastic constant from Eq. (21) or Eq. (23).

The bulk modulus $B$ is obtained from the volume-dependent energy of the undistorted crystal by

$$
B=V \frac{d^{2} E}{d V^{2}}
$$

The energy was evaluated at volume intervals of $5 \%$ of the normal volume, from $20 \%$ expanded to $50 \%$ contracted. Derivatives were evaluated by fitting the equation of state of Rose et al. [69 to the energies and differentiating the function. It was found that a single curve of this type did not accuaretly fit both the high density points and the points near the minimum, so seperate overlapping fits were made for the 10 highest and lowest densities.

The electronic structure calculations were based on the linearized augmented planewave (LAPW) code WIEN97 [70]. The energy functional used was the generalizedgradient approximation as parameterized by Perdew, Burke, and Ernzerhof [71]. Some numerical parameters used in the calculations for $\mathrm{Cu}(\mathrm{Au})$ were, in atomic units: muffin tin radius $r_{\mathrm{MT}}=1.8(2.0)$, plane wave cut-off $r_{\mathrm{MT}} k_{\max }=9.0$, cut-off for expansion of density and potential $g_{\max }=16(19)$; Brillouin zone integrals used special points corresponding to $16^{3}\left(18^{3}\right)$ points in the full zone, with Gaussian smearing of the energies by 20 mRy.

Our results on $C^{\prime}, C_{44}$ and $B$ for $\mathrm{Cu}$ and $\mathrm{Au}$ are shown in Tables 5 and 6 , respectively. It is interesting to note the increasing anisotropy of Au under pressure. From Table 6 we see that, for $\mathrm{Au}, C^{\prime}$ does not increase nearly as rapidly as $C_{44}$ with compression. This is connected with the fact that the energy difference between the fcc and bcc structures is small at all pressures [72]. The distortion corresponding to $C^{\prime}$ is along the Bain path connecting fcc to bcc, and it has been seen 68 that a small energy difference between these structures correlates with a small value of $C^{\prime}$.

Let us now turn to the calculation of the shear moduli of $\mathrm{Cu}$ and $\mathrm{Au}$. For a solid of cubic crystalline structure, as analysis by Kröner 28 shows, successively narrower bounds can be placed on the shear modulus as the degree of disorder in grain orientation increases. In the limit of perfect disorder, the value of the shear modulus is the single positive real root of a cubic equation with coefficients that depend on the single-crystal elastic constants $C^{\prime}, C_{44}$, and $B$ :

$$
x^{3}+\frac{9 B+4 C^{\prime}}{8} x^{2}-\frac{3\left(B+4 C^{\prime}\right) C_{44}}{8} x-\frac{3 B C^{\prime} C_{44}}{4}=0 .
$$

The values of the shear modulus calculated from Eq. (25) are shown in Tables 5 and 6 along with $C^{\prime}, C_{44}$ and $B$.

As a by-product of our analysis, we obtain the interesting results that $G\left(2 \rho_{0}, 0\right) \simeq$ $10 G\left(\rho_{0}, 0\right)$ for $\mathrm{Cu}$, and $G\left(2 \rho_{0}, 0\right) \simeq 20 G\left(\rho_{0}, 0\right)$ for $\mathrm{Au}$.

In Figs. 7 and 8 we compare Eqs. (18) and (19) with $T=0$, for $\mathrm{Cu}$ and $\mathrm{Au}$, to the corresponding $G$ entries in Tables 5 and 6 . 


\begin{tabular}{|c|c|c|c|c|}
\hline$\rho, \mathrm{g} / \mathrm{cc}$ & $C^{\prime}, \mathrm{GPa}$ & $C_{44}, \mathrm{GPa}$ & $B, \mathrm{GPa}$ & $G, \mathrm{GPa}$ \\
\hline 8.850 & 30.404 & 77.639 & 142.15 & 53.912 \\
9.833 & 41.863 & 124.78 & 235.73 & 81.901 \\
11.06 & 48.599 & 167.42 & 386.02 & 104.66 \\
12.64 & 83.020 & 260.81 & 652.48 & 168.57 \\
14.75 & 130.48 & 445.26 & 1151.8 & 279.70 \\
17.70 & 229.71 & 800.23 & 2118.4 & 499.25 \\
\hline
\end{tabular}

Table 5. The single-crystal elastic constants and shear modulus of $\mathrm{Cu}$ as functions of density from the electronic-structure calculations described in the text.

\begin{tabular}{|c|c|c|c|c|}
\hline$\rho, \mathrm{g} / \mathrm{cc}$ & $C^{\prime}, \mathrm{GPa}$ & $C_{44}, \mathrm{GPa}$ & $B, \mathrm{GPa}$ & $G, \mathrm{GPa}$ \\
\hline 19.29 & 16.445 & 31.690 & 201.20 & 24.585 \\
21.43 & 19.550 & 77.940 & 339.57 & 46.764 \\
24.11 & 33.890 & 127.75 & 568.39 & 78.093 \\
27.56 & 35.053 & 255.22 & 1029.0 & 127.48 \\
32.15 & 69.837 & 479.27 & 1918.2 & 243.34 \\
38.58 & 121.16 & 912.15 & 3753.2 & 451.65 \\
\hline
\end{tabular}

Table 6. The single-crystal elastic constants and shear modulus of Au as functions of density from the electronic-structure calculations described in the text.

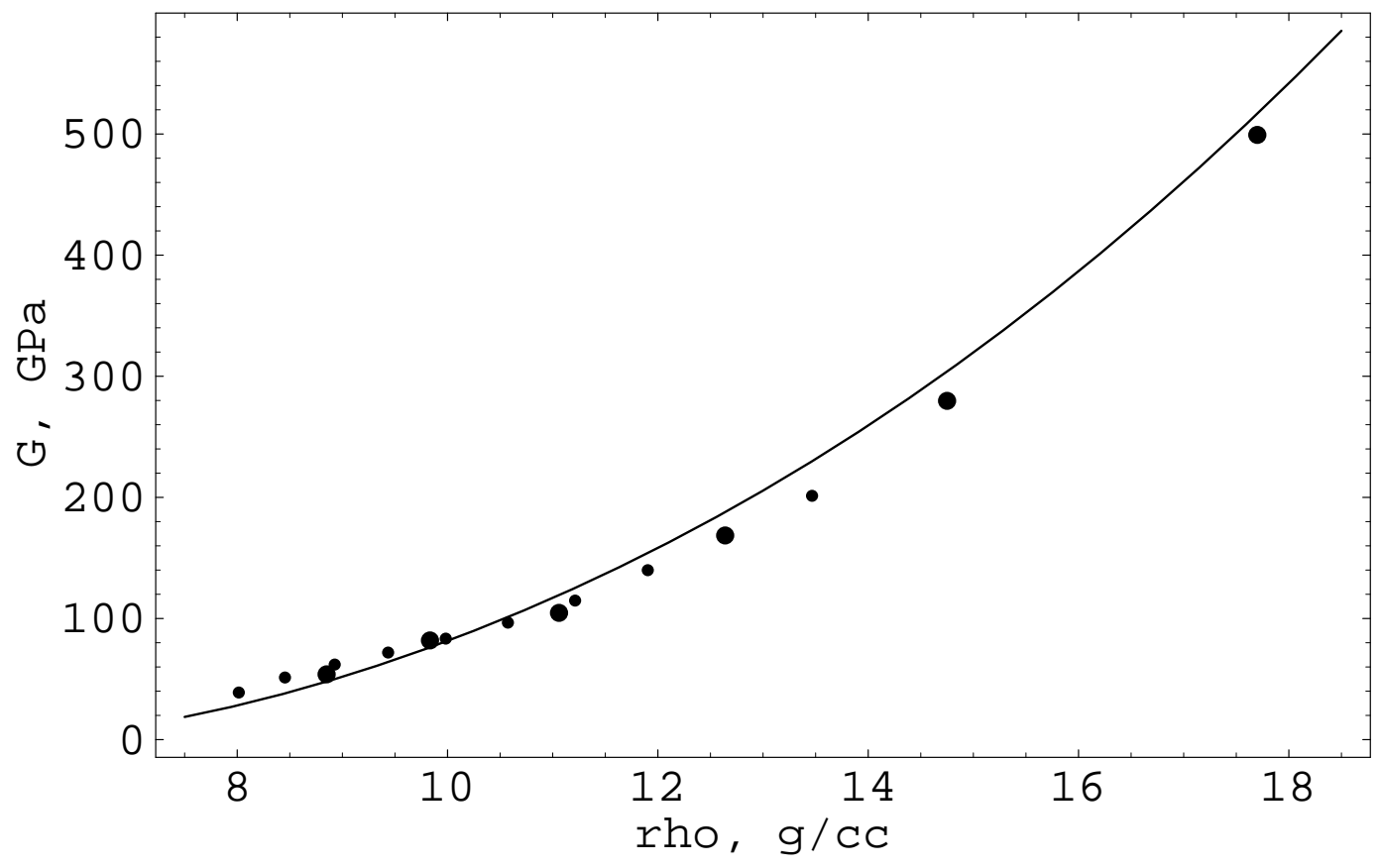

Fig. 7. The $T=0$ shear modulus of Cu: Eq. (18) vs. electronic-structure calculations (larger points, Table 5). The smaller points, obtained from first-principles calculations [26], are shown for comparison. 


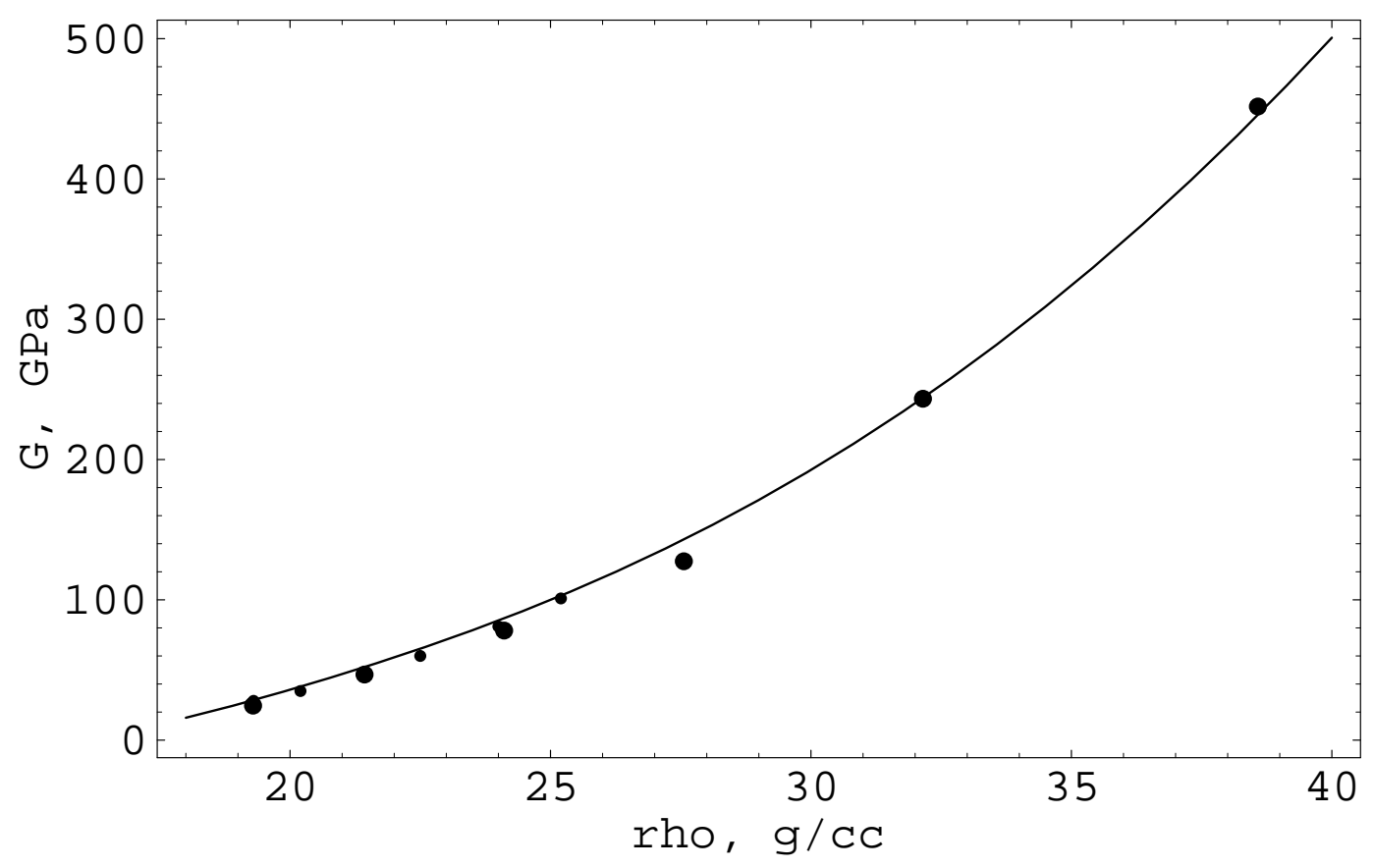

Fig. 8. The $T=0$ shear modulus of Au: Eq. (19) vs. electronic-structure calculations (larger points, Table 6). The smaller points, obtained from first-principles calculations [73], are shown for comparison.

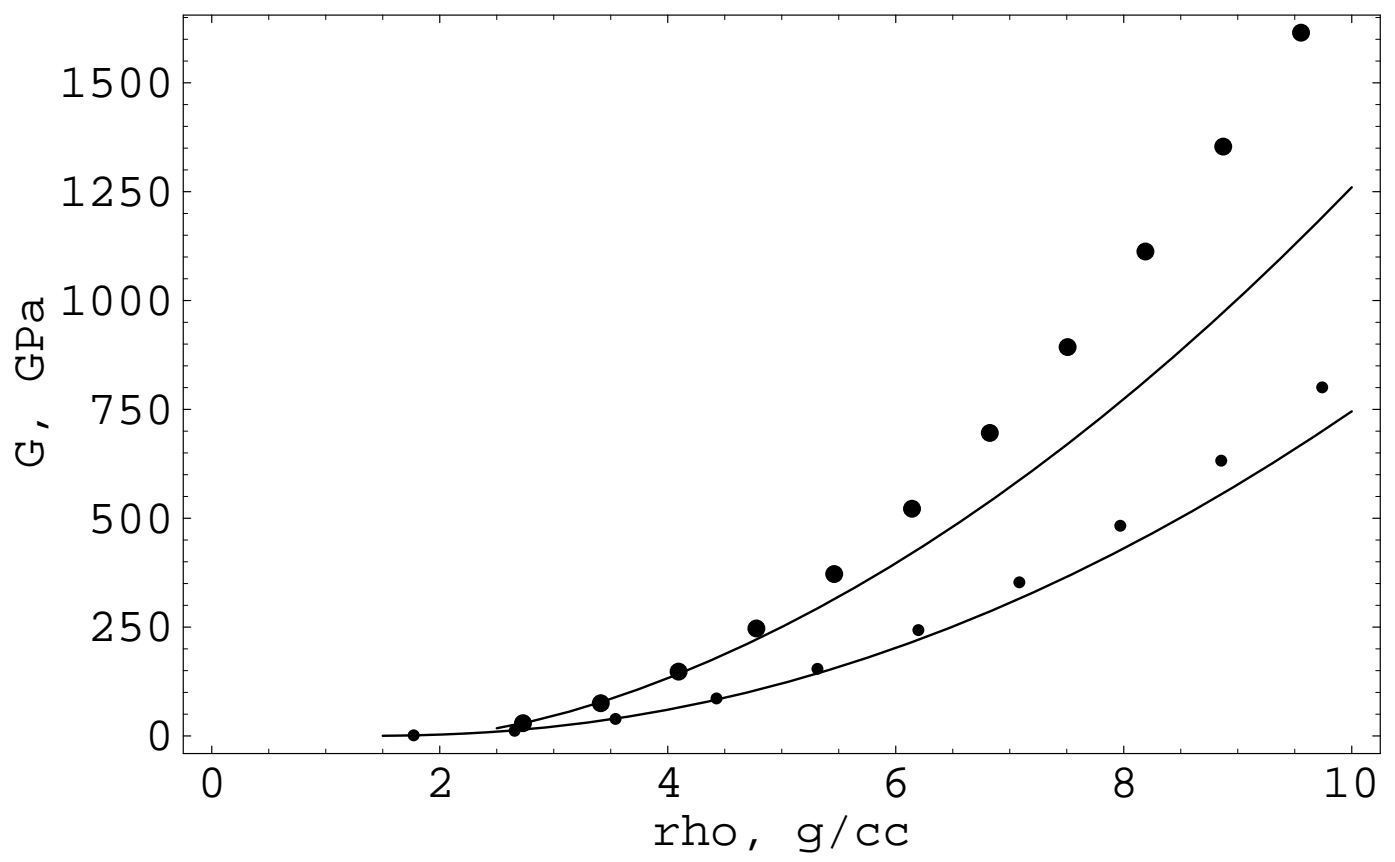

Fig. 9. Comparison of the two models for the $T=0$ shear modulus: Eqs. (16) and (17) vs. the corresponding Guinan-Steinberg values for Ar (smaller points) and Al (larger points). 
Finally, it is interesting to compare our model at $T=0$ to the Guinan-Steinberg model mentioned in the Introduction. The equation of state, $P=P(\rho)$, is needed to make this comparison. In Fig. 9 the models are compared to each other for $\mathrm{Ar}$ and $\mathrm{Al}$. The corresponding equations of state are taken from ref. [74]. For Al, $G_{0}^{\prime}=1.8$ comes from ref. [75]. For Ar, $G_{0}^{\prime}=1.6$ is obtained from the relation $445 \gamma_{0}=B_{0} / 2 G_{0}^{\prime} / G_{0}-1 / 6$ with $\gamma_{0}=\gamma\left(\rho_{0}\right)$ from Eq. (11) and $B_{0}$ taken from ref. [74]. The values of $\rho_{0}$ and $G_{0}=G\left(\rho_{0}, 0\right)$ can be found in Table 4.

It is seen that agreement between the two models is good at low densities, but it gradually deteriorates with increasing compression. The reason for this must be that the Guinan-Steinberg model generally provides only the correct functional form $G \sim \rho^{4 / 3}$ in the limit of infinite compression, not the precise numerical value of $G$ in that limit, in contrast to our model which provides both.

\section{Concluding remarks}

We have constructed an analytic model of the shear modulus applicable at all densities greater than or of order ambient $(G(\rho, 0) \rightarrow 0$ as $\rho \rightarrow 0$, as required, but the model may not be quantitatively correct for expanded states), and temperatures from 0 to $T_{m}$. All of the model parameters can be obtained from low-pressure experimental data. The model has the proper low-pressure and high-pressure limits, by construction, and to within uncertainties it agrees with electronic-structure values of $G$ for $\mathrm{Cu}$ and $\mathrm{Au}$ to compressions of 2, which roughly corresponds to pressures of $5 \mathrm{Mbar}$ for $\mathrm{Cu}$ and $9 \mathrm{Mbar}$ for $\mathrm{Au}$.

The above comparisons of our shear modulus model, which includes a model for $T_{m}(\rho)$, to electronic structure calculations and experimental data on $\mathrm{Ar}, \mathrm{Al}, \mathrm{Cu}$, and $\mathrm{Au}$ show very good agreement. This suggests that our model accurately represents the density and temperature dependence of the shear moduli of monatomic solids in general. There is, however, no theoretical justification for applying our model to alloys or compounds, although in practice it may work reasonably well in these cases. Its generalization to more complex materials would involve generalizing our model for the Grüneisen parameter. A functional form for $\gamma(\rho)$ depends critically on the asymptotic $(\rho \rightarrow \infty)$ form of the equation of state [34, and it has been suggested that the asymptotic forms of the equations of state of more complex materials, e.g., ionic, covalent, or molecular crystals, are different from that of a metal[76]. If so, the limiting value of $\gamma$ is unknown (not necessarily 1/2) for such materials. In that case, an analytic model for the Grüneisen parameter cannot be constructed, hence analytic forms for the melting curve and shear modulus cannot be obtained.

We now briefly discuss three potential applications of our model.

(1) Plastic deformation of metals at high pressure. It is generally assumed that the ratio of the plastic flow stress (shear stress necessary to induce plastic deformation at a given strain rate) to the shear modulus is approximately independent of pressure. In other words, the predominant pressure dependence of the plastic flow stress is contained in the shear modulus. An accurate, simple analytic (for fast evaluation) model of the shear modulus is therefore essential for numerical simulations of material deformation over extremes in pressure. 
(2) Numerical simulations of elastic wave propagation, including pressure release waves in shocked solids. The differential stress deviator, $d s_{i j}$, is equal to $2 G(\rho, T)\left(d \epsilon_{i j}-\right.$ $\left.\delta_{i j} d \epsilon_{k k} / 3\right)$ plus material rotation terms $\left(d \epsilon_{i j}\right.$ is the differential elastic strain), thus a model of the shear modulus is required for calculations of elastic wave propagation in materials with sufficiently high yield stresses that the stress deviators are not negligible. The speed of a release wave in a shocked solid depends on $G\left(\rho_{H}, T_{H}\right)$, where $\rho_{H}$ and $T_{H}$ are the density and temperature of the shocked state.

(3) Pulsations and quakes of dense stars. Hansen and Van Horn [77] have done a preliminary analysis of the effects of crystalline cores on the oscillations of white dwarfs and found that the $g$-like spheroidal mode frequencies are increased by approximately a factor of two, concluding that the elastic shear strength of the core must be taken into account in the computation of cool white dwarf oscillations. The inclusion of elastic shear strength in the neutron star pulsation equations of McDermott et al. [78] resulted in the appearance of two classes of oscillation modes not present in a fluid neutron star. The change in the shape of the surface following a neutron star quake is proportional to the shear modulus of the crust [79].

Further tests of our model for the shear modulus should be made as high-pressure experimental data and electronic structure results become available for elements other than argon, aluminum, copper and gold. New zero-pressure data are also needed to generate additional sets of model parameters.

\section{Acknowledgements}

We wish to thank J.C. Boettger, J.D. Johnson and G.W. Pfeufer for very stimulating discussions on the subject of the shear modulus.

\section{References}

[1] A. Wolfenden and M.R. Harmouche, J. Mat. Sci. 28 (1993) 1015

[2] S.M. Collard and R.B. McLellan, Acta Metal. Mater. 39 (1991) 3143

[3] D. Vidal, Compt. Rend. Acad. Sci., Ser. B, 279 (1974) 251

[4] E. Walker, Solid State Comm. 28 (1978) 587

[5] D. Gerlich and E.S. Fisher, J. Phys. Chem. Sol. 30 (1969) 1197; J.L. Tallon and A. Wolfenden, ibid., 40 (1979) 831

[6] H.R. Moeller and C.F. Squire, Phys. Rev. 151 (1966) 689; G.J. Keeler and D.N. Batchelder, J. Phys. C 3 (1970) 510

[7] E.W. Kammer et al., J. Phys. Chem. Sol. 33 (1972) 1891

[8] Y.A. Chang and L. Himmel, J. Appl. Phys. 37 (1966) 3787

[9] N. Nücker and U. Buchenau, Phys. Rev. B 31 (1985) 5479 
[10] A. Larose and B.N. Brockhouse, Can. J. Phys. 54 (1976) 1990

[11] C.L. Vold et al., J. Phys. Chem. Sol. 38 (1977) 157

[12] G. Fritsch and H. Bube, Phys. Stat. Sol. A 30 (1975) 571

[13] G. Fritsch, F. Geipel and A. Prasetyo, J. Phys. Chem. Sol. 34 (1973) 1961

[14] Y. Talmor, E. Walker and S. Steinemann, Solid State Comm. 23 (1977) 649

[15] R. Balzer, D.S. Kupperman and R.O. Simmons, Phys. Rev. B 4 (1971) 3636

[16] E. Walker and P. Bujard, Solid State Comm. 34 (1980) 691

[17] A.A. Valiev, S.P. Nikanorov and A.V. Stepanov, Fiz. Tv. Tela 12 (1970) 1656 [Sov. Phys. Solid State 12 (1970) 1312]

[18] N.A. Lurie, G. Shirane and J. Skalyo, Phys. Rev. B 9 (1974) 2660

[19] G.A. Alers and J.R. Neighbours, J. Phys. Chem. Sol. 7 (1958) 58

[20] G.K. Straub, J.M. Wills and D.C. Wallace, in Shock Waves in Condensed Matter 1987, Eds. S.C. Schmidt and N.C. Holmes, (North-Holland, Amsterdam, 1988), p. 207

[21] N.E. Christensen, A.L. Ruoff and C.O. Rodriguez, Phys. Rev. B 52 (1995) 9121

A.L. Ruoff, C.O. Rodriguez and N.E. Christensen, Phys. Rev. B 58 (1998) 2998

[22] P. Söderlind, J.A. Moriarty and J.M. Wills, Phys. Rev. B 53 (1996) 14063

[23] P. Söderlind and J.A. Moriarty, Phys. Rev. B 57 (1998) 10340

[24] S.K. Schiferl and D.C. Wallace, Phys. Rev. B 31 (1985) 7662

[25] C.W. Greeff and J.A. Moriarty, Phys. Rev. B 59 (1999) 3427

[26] S.P. Rudin, M.D. Jones, C.W. Greeff and R.C. Albers, Phys. Rev. B 65 (2002) 235114

[27] J.P. Watt, J. Appl. Phys. 50 (1979) 6290, 51 (1980) 1520, 60 (1986) 3120; J.P. Watt and L. Peselnick, J. Appl. Phys. 51 (1980) 1525

[28] E. Kröner, Z. Phys. 151 (1958) 504; J. Eng. Mech. Div. ASCE 108 (1980) 899, correction - ibid., 107 (1981) 1249

[29] M. Guinan and D. Steinberg, J. Phys. Chem. Sol. 36 (1975) 829

[30] D.L. Preston and D.C. Wallace, Solid State Comm. 81 (1992) 277

[31] O.L. Anderson, Equations of State of Solids for Geophysics and Ceramic Science, (Oxford University Press, Oxford, 1995), Chapter 9

[32] L. Burakovsky and D.L. Preston, Solid State Comm. 115 (2000) 341 
[33] L. Burakovsky, D.L. Preston and R.R. Silbar, Phys. Rev. B 61 (2000) 15011

[34] L. Burakovsky and D.L. Preston, An analytic model for the Grüneisen parameter at all densities, Los Alamos preprint LA-UR-02-2690, submitted for publication

[35] D.A. Young, Phase Diagrams of the Elements, (Univ. Calif. Press, Berkeley, 1991)

[36] O.K. Belousov, Izvestiya Rossiiskoi Akademii Nauk. Metally, No.3 (1993) 29 [Russian Metallurgy, No.3 (1993) 25]

[37] L. Burakovsky and D.L. Preston, Phys. Rev. E 63 (2001) 067402

[38] H. DeWitt and W. Slattery, Contrib. Plasma Phys. 39 (1999) 97; H. DeWitt et al., ibid. 41 (2001) 251

[39] A.Y. Potekhin and G. Chabrier, Phys. Rev. E 62 (2000) 8554

[40] H.L. Helfer, R.L. McCory and H.M. Van Horn, J. Stat. Phys. 37 (1984) 577

[41] F.H. Ree, in Molecular Systems Under High Pressure, Eds. R. Pucci and G. Piccitto, (North-Holland, Amsterdam, 1991), p. 33

[42] S. Ogata and S. Ichimaru, Phys. Rev. A 42 (1990) 4867

[43] P. Sisodia, A. Dhoble and M.P. Verma, Phys. Stat. Sol. B 163 (1991) 345

[44] V.P. Kopyshev, Doklady Akad. Sci. USSR 161 (1965) 1067 [Sov. Phys. Doklady 10 (1965) 338]

[45] L. Burakovsky, D.L. Preston and R.R. Silbar, J. Appl. Phys. 88 (2000) 6294

[46] H. Ledbetter, Z. Metallk. 82 (1991) 820

[47] H. Siethoff and K. Ahlborn, Phys. Stat. Sol. B 190 (1995) 179

[48] H. Siethoff, Phys. Stat. Sol. B 200 (1997) 57

[49] H. Siethoff, Phys. Stat. Sol. B 222 (2000) 25

[50] H. Siethoff and K. Ahlborn, J. Appl. Phys. 79 (1996) 2968

[51] H. Siethoff, Intermetallics 5 (1997) 625

[52] K. Ishizaki, I.L. Spain and P. Bolsaitis, J. Phys. Chem. Sol. 63 (1975) 1401

[53] V.M. Cheng, W.B. Daniels and R.K. Crawford, Phys. Lett. A 43 (1973) 109

[54] V.E. Zinov'ev, Handbook of Thermodynamical Properties of Metals at High Temperatures, (Nova Science Publishers, New York, 1996)

[55] E.A. Kraut and G.C. Kennedy, Phys. Rev. Lett. 16 (1966) 608, Phys. Rev. 151 (1966) 668 
[56] B. Strauss et al., Phys. Rev. B 33 (1986) 7852

[57] C.-S. Zha et al., J. Chem. Phys. 85 (1986) 1034

[58] A. Hänström and P. Lazor, J. Alloys Comp. 305 (2000) 209

[59] J.A. Moriarty, D.A. Young and M. Ross, Phys. Rev. B 30 (1984) 578

[60] J.D. Johnson, private communication

[61] V.D. Urlin, Zh. Eksp. Teor. Fiz. 49 (1965) 485 [Sov. Phys. JETP 22 (1966) 341]

[62] J.A. Moriarty, in Shock Waves in Condensed Matter, Ed. Y.M. Gupta, (Plenum Press, New York, 1986), p. 101

[63] D.B. Hayes, R.S. Hixson and R.G. McQueen, in Shock Compression of Condensed Matter - 1999, Eds. M.D. Furnish, L.C. Chhabildas and R.S. Hixson, (AIP, Melville, NY, 2000), p. 483

[64] M. Grimsditch, P. Loubeyre and A. Polian, Phys. Rev. B 33 (1986) 7192

[65] H. Shimizu et al., Phys. Rev. Lett. 86 (2001) 4568

[66] G.V. Sin'ko and N.A. Smirnov, J. Phys. Cond. Mat. 14 (2002) 6989

[67] M.J. Mehl, B.M. Klein and D.A. Papaconstantopoulos, in Intermetallic Compounds: Priciples and Practice, Eds. J.H. Westbrook and R.L. Fleischer, (Wiley, London, 1995), Vol. I

[68] P. Söderlind, O. Eriksson, J.M. Wills and A.M. Boring, Phys. Rev. B 48 (1993) 5844

[69] J.H. Rose, J.R. Smith, F. Guinea, and J. Ferrante, Phys. Rev. B 29 (1984) 2963

[70] P. Blaha, K. Schwarz and J. Luitz, WIEN97, Vienna University of Technology, 1997 (the improved and updated Unix version of the original copyrighted WIEN-code, which was published by P. Blaha et al. in Comput. Phys. Comm. 59 (1990) 399)

[71] J.P. Perdew, S. Burke and M. Ernzerhof, Phys. Rev. Lett. 77 (1996) 3865

[72] J.C. Boettger, private communication

[73] T. Tsuchiya and K. Kawamura, J. Chem. Phys. 116 (2002) 2121

[74] J. Hama and K. Suito, J. Phys. Cond. Mat. 8 (1996) 67

[75] M.W. Guinan and D.J. Steinberg, J. Phys. Chem. Sol. 35 (1974) 1501

[76] B.I. Davydov, Bull. Acad. Sci. USSR, Geophys. Ser. 12 (1956) 1411

[77] C.J. Hansen and H.M. Van Horn, Ap. J. 233 (1979) 253

[78] P.N. McDermott, H.M. Van Horn, and C.J. Hansen, Astrophys. J. 325 (1988) 72

[79] M. Ruderman, Nature 233 (1969) 597 